Arq. Bras. Med. Vet. Zootec., v.56, n.3, p.300-306, 2004

\title{
Presença de Salmonella sp. no trato intestinal e em tonsilas/linfonodos submandibulares de suínos ao abate
}

[Presence of Salmonella sp. in the intestinal tract and tonsils/mandibular lymph nodes in pigs at slaughter]

\author{
S.M.F. Castagna, P. Schwarz, C.W. Canal, M. Cardoso* \\ ${ }^{1}$ Faculdade de Veterinária, Universidade Federal do Rio Grande do Sul \\ Av. Bento Gonçalves, 9090 \\ 91450-000 - Porto Alegre, RS
}

\begin{abstract}
RESUMO
Estudou-se a associação entre o isolamento de Salmonella sp. do trato intestinal de suínos ao abate e sua presença em tonsilas/linfonodos submandibulares (T/LS), utilizadas no preparo de embutidos. Colheramse linfonodos mesentéricos (LM), conteúdo intestinal (CI) e T/LS de 90 animais. Em 71 (79\%) deles isolou-se Salmonella sp. em pelo menos uma das amostras coletadas. Observou-se associação entre amostras de T/LS positivas e isolamento de Salmonella sp. a partir de LM e/ou CI ( $\mathrm{P}<0,001)$, com odds ratio $_{\text {LTpositivo }} 5,6$. T/LS de outros 75 animais foram processados separadamente, verificando-se associação $(\mathrm{P}<0,0001)$ entre T/LS e isolamento de Salmonella. Os sorovares mais encontrados foram Typhimurium e Bredeney. Conclui-se que a presença de Salmonella sp. no trato gastrintestinal é fator de risco para a contaminação de partes da carcaça utilizadas na fabricação de embutidos.
\end{abstract}

Palavras-chave: suíno, Salmonella sp., linfonodos submandibulares, tonsilas, trato intestinal

\begin{abstract}
The association between Salmonella $s p$. isolated from the intestinal tract of pigs at slaughter and its presence in tonsils/mandibular lymph nodes (T/MDL) used in the sausage manufacture was assessed. In a trial mesenteric lymph nodes (LM), intestinal contents (IC) and T/MDL were collected from 90 animals. Salmonella sp. was isolated from 71 (79\%) animals in at least one of the samples. T/MDL contaminated samples were correlated with Salmonella $s p$. isolated from $L M$ and/or IC $(P<0.001)$, with odds ratio $_{\text {LTpositive }}$ 5.6. In other trial, T/MDL of 75 animals processed separately, were associated with isolation of Salmonella $s p(P<0.0001)$. The serovars more prevalent were Typhimurium and Bredeney. It was concluded that Salmonella $s p$. presence in the intestinal tract is a risk factor for contamination of the portions of carcasses used in sausage manufacture.
\end{abstract}

Keywords: swine, Salmonella sp., mandibular lymph node, tonsils, intestinal tract

\section{INTRODUÇÃO}

A salmonelose é uma das principais doenças transmitidas por alimentos de origem animal de maior impacto na saúde pública. Os surtos relatados, geralmente, estão associados ao consumo de ovos e carne de frango, porém, nos últimos anos, tem aumentado o número de casos de salmonelose humana relacionados ao consumo de produtos de origem suína (FedorkaCray, 1996). Suínos infectados pela grande maioria dos sorovares de Salmonella não exibem sinais clínicos da doença, sendo, porém,

Recebido para publicação em 4 de julho de 2003

Recebido para publicação, após modificações, em 4 de fevereiro de 2004

*Autor para correspondência.

E-mail: mcardoso@vortex.ufrgs.br 
portadores que podem excretar a bactéria de forma intermitente.

Em estudo prévio realizado no Rio Grande do Sul, a prevalência de suínos portadores assintomáticos de Salmonella sp. em linfonodos mesentéricos e conteúdo intestinal, no momento do abate, foi de 55,6\% (Bessa et al., 2001). No mesmo estudo, observou-se variação entre 50 e $61,3 \%$ na prevalência encontrada em diferentes matadouros. Linfonodos mesentéricos e conteúdo intestinal positivos podem ser responsáveis pela contaminação cruzada de carcaças durante o processamento, mas não são produtos que chegam até o consumidor. Tonsilas e linfonodos submandibulares permanecem na carcaça após o abate e, juntamente com músculos da região da cabeça, são empregados na fabricação de embutidos e carne mecanicamente separada. Caso estejam contaminados, esses ingredientes podem ser diretamente responsáveis pela presença de Salmonella nesses produtos finais. O objetivo do presente estudo foi avaliar a associação entre a presença de Salmonella sp. no trato gastrintestinal e em linfonodos submandibulares e tonsilas de suínos ao abate.

\section{MATERIAL E MÉTODOS}

O estudo foi conduzido em um estabelecimento com Serviço de Inspeção Federal, localizado no Estado do Rio Grande do Sul, onde havia sido encontrada alta prevalência de suínos positivos para Salmonella sp. em estudo anterior (Bessa et al., 2001). O experimento foi dividido em duas etapas. A etapa 1 ocorreu entre setembro de 2000 e fevereiro de 2001, em seis visitas ao frigorífico. Em cada visita, foram coletados linfonodos mesentéricos (LM), fragmento (conteúdo) de intestinos (CI) e um pool de linfonodos submandibulares e tonsilas (LT) de 15 animais. A primeira carcaça foi selecionada ao acaso e as demais foram escolhidas em intervalo calculado a partir do número previsto de animais a serem abatidos pelo número de animais a serem coletados. As amostras coletadas foram transportadas em sacos plásticos individuais sob refrigeração. No laboratório, cada amostra $(25 \mathrm{~g})$ foi submetida a protocolo de isolamento descrito por Michael et al. (1999). As colônias típicas e suspeitas de Salmonella sp. foram identificadas segundo suas características morfológicas e bioquímicas, conforme metodologia de rotina (Holt et al., 1994). As bactérias presuntivamente identificadas como
Salmonella foram testadas com soro polivalente ${ }^{1}$. Quando positivas, as amostras foram encaminhadas ao Instituto Oswaldo Cruz, no Rio de Janeiro, para sorotipificação definitiva. A etapa 2 ocorreu entre março e julho de 2001, em três visitas realizadas. Em cada visita, linfonodos submandibulares (LS) e tonsilas (T) foram coletados de carcaças de 25 animais, sendo processados separadamente no laboratório. O protocolo de isolamento de Salmonella sp. foi o mesmo utilizado na etapa anterior, apenas com os volumes adaptados para $10 \mathrm{~g}$ de amostra, de forma que a diluição inicial (1:10) fosse mantida. Todas as amostras de Salmonella sp., isoladas e estocadas a $-20^{\circ} \mathrm{C}$ em caldo infusão cérebro e coração (BHI) acrescido de glicerol a $20 \%$, foram testadas quanto à sua resistência aos antimicrobianos pelo método da difusão em ágar Mueller-Hinton (Barry e Thornsberry, 1985). No teste foram utilizados $\operatorname{discos}^{2}$ dos seguintes antimicrobianos: ácido nalidíxico $(30 \mu \mathrm{g}), \quad$ amicacina $(30 \mu \mathrm{g})$, amoxacilina/ácido clavulânico (20/10 $\mu \mathrm{g})$, ampicilina $(10 \mu \mathrm{g})$, cefaclor $(30 \mu \mathrm{g})$, ciprofloxacina $(5 \mu \mathrm{g})$, cloranfenicol $(30 \mu \mathrm{g})$, cotrimoxazol $(5 \mu \mathrm{g})$, estreptomicina $(10 \mu \mathrm{g})$, gentamicina $(10 \mu \mathrm{g})$, neomicina $(30 \mu \mathrm{g}), \quad$ sulfonamida $(300 \mu \mathrm{g})$, tetraciclina $(30 \mu \mathrm{g})$ e tobramicina $(10 \mu \mathrm{g})$. Os resultados da primeira etapa e do perfil de resistência frente aos antimicrobianos foram avaliados por meio do teste do qui-quadrado e os da segunda etapa, pelo teste exato de Fisher, ambos do pacote estatístico SAS (User's..., 1999). Para o cálculo do odds ratio foi utilizado o intervalo de confiança de $95 \%$.

\section{RESULTADOS E DISCUSSÃO}

$\mathrm{Na}$ etapa 1 foi isolada Salmonella sp. de pelo menos uma das amostras analisadas de 71 (79\%) dos 90 animais coletados (Tab. 1). Os maiores índices de isolamento de Salmonella foram obtidos a partir de LM (55/90; 61\%), seguido de CI $(50 / 90 ; 55,5 \%)$ e LT $(33 / 90 ; 36,7 \%)$. A alta prevalência de suínos portadores de Salmonella sp. confirma os resultados encontrados previamente no mesmo abatedouro por Bessa et al. (2001), reforçando relatos anteriores sobre a persistência da infecção por Salmonella nos sistemas de produção (Blaha, 2001).

\footnotetext{
${ }^{1}$ Soro Salmonella Polivalente, PROBAC, São Paulo, Brasil.

${ }^{2}$ Sensidisc, DME, Araçatuba, Brasil.
} 
Castagna et al.

Tabela 1. Número de amostras positivas para Salmonella $\mathrm{sp}$. em linfonodos submandibulares/tonsilas (LT), linfonodos mesentéricos (LM) e conteúdo intestinal (CI) de suínos ao abate, segundo a coleta

\begin{tabular}{lcccccccc}
\hline \multirow{2}{*}{ Coleta } & Animais & LT & LM & CI & LT+LM+CI & LT+LM & LT+CI & LM+CI \\
\cline { 2 - 8 } & 15 & 0 & 1 & 2 & 1 & 0 & 1 & 2 \\
I & 15 & 1 & 2 & 1 & 6 & 1 & 0 & 2 \\
II & 15 & 1 & 1 & 1 & 2 & 1 & 0 & 4 \\
III & 15 & 0 & 5 & 4 & 1 & 0 & 0 & 2 \\
IV & 15 & 1 & 3 & 0 & 5 & 0 & 1 & 5 \\
V & 15 & 0 & 2 & 1 & 7 & 2 & 2 & 0 \\
VI & 90 & 3 & 14 & 9 & 22 & 4 & 4 & 15 \\
Total & & & & & & & &
\end{tabular}

Suínos portadores assintomáticos de Salmonella sp. no trato intestinal e linfonodos mesentéricos podem ser importante fonte de contaminação cruzada na linha de abate (Di Guardo et al., 1992). Apesar da importância do CI e dos LM na identificação de animais portadores, esses tecidos são retirados da carcaça durante $o$ processamento, não sendo produtos de consumo humano. Dessa forma, apesar do índice de isolamento encontrado em LT ter sido inferior aos encontrados para CI e LM, representam maior risco ao consumidor pois, juntamente com músculos da região da cabeça, são utilizados na fabricação de embutidos e carne mecanicamente separada. Esses produtos, caso não sofram tratamento térmico na indústria, podem chegar contaminados ao consumidor, representando considerável risco para a ocorrência de infecção alimentar.

Em amostras coletadas de uma mesma carcaça observou-se que, dos 68 suínos positivos em LM e/ou CI, 30 animais $(44,1 \%)$ foram positivos também em LT, enquanto 22 (31\%) foram positivos nas três amostras coletadas. Em três animais $(4,2 \%)$ foi isolada Salmonella sp. somente de LT.

Observou-se associação entre amostras LT positivas para Salmonella sp. e amostras LM e/ou CI positivas $(\mathrm{P}=0,001)$, com odds ratio (OR) LT positivo 5,6 (intervalo de confiança: 2,2 14,2). A presença de Salmonella no conteúdo intestinal foi considerada por Berends et al. (1997) como a principal determinante da contaminação de carcaças durante o processamento. Segundo os autores, a presença da bactéria no intestino como fator de risco para carcaças positivas apresentou OR de 3,5, estimando-se que $70 \%$ das carcaças positivas ao final da linha de abate resultaram de contaminação proveniente do próprio animal portador.

Observou-se associação entre CI positivo e presença de Salmonella sp. em LM $(\mathrm{P}=0,001)$, e entre LM e CI positivos $(\mathrm{P}=0,01)$, com

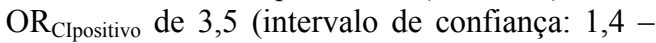
$8,4)$. Do total de animais positivos, $37(54,4 \%)$ apresentavam Salmonella sp. em LM e CI, 18 $(26,5 \%)$ foram isolados apenas em LM e 13 $(19,1 \%)$ somente no CI. Esse perfil de isolamento está de acordo com as diversas situações que podem ocorrer após a infecção, portador, com ou sem excreção do agente, ou disseminador passivo.

Alguns estudos têm apontado suínos positivos em linfonodos mesentéricos como sendo portadores assintomáticos de infecção prévia, ocorrida na granja. Estes animais, principalmente pelo estresse durante o transporte até o abatedouro, poderiam excretar Salmonella sp. nas fezes, contaminando outros suínos, que passariam a ser positivos no conteúdo intestinal e nas tonsilas, tornando-se disseminadores passivos (Morrow et al., 2000). O risco potencial desses animais durante o processamento foi demonstrado por Swanenburg et al. (2001a), que encontraram correlação significativa entre presença de Salmonella sp. em tonsilas e conteúdo intestinal com carcaças positivas na linha de abate.

Estudos recentes (Swanenburg et al., 2001b; Rostagno et al., 2002) comprovaram a influência do tempo de permanência em currais pré-abate com alto nível de contaminação residual por Salmonella sobre o número de suínos positivos ao abate. Hurd et al. (2001) verificaram que, sob condições experimentais, a Salmonella sp. pode infectar suínos expostos a ambientes contaminados após duas horas de contato. 
Dessa forma, onde se constataram índices de isolamento em linfonodos mesentéricos e submandibulares, seria possível supor que, na maioria dos casos, os animais teriam sido contaminados na granja. Entretanto, o tempo de descanso médio observado nesse frigorífico foi superior a seis horas, tempo suficiente para que ocorresse a infecção de suínos que chegassem negativos para o abate.

Considerando que na primeira etapa a amostra LT foi composta de linfonodos submandibulares e tonsilas, na segunda etapa buscou-se verificar qual desses componentes foi responsável pela presença de Salmonella na amostra. Em 30 animais $(40 \%)$, dos 75 submetidos à coleta, foi possível isolar Salmonella sp. de pelo menos um dos materiais analisados (Tab. 2). Este índice de isolamento não difere do encontrado na primeira etapa para LT $(36,7 \%)$. O isolamento a partir de T $(27 / 30 ; 90,0 \%)$ foi maior do que o de LS $(23 / 30 ; 77,0 \%)$. Em $20(66,7 \%)$ dos animais positivos foi isolado Salmonella sp. simultaneamente, de LS e T.

Tabela 2. Número de amostras positivas para Salmonella sp. isoladas de linfonodos submandibulares (LS) e tonsilas (T) de suínos ao abate, segundo a coleta

\begin{tabular}{lccccc}
\hline Coleta & Animais & $\begin{array}{c}\text { Total de } \\
\text { positivos }\end{array}$ & LS & T & LS+T \\
\hline I & 25 & 4 & 2 & 2 & 0 \\
II & 25 & 21 & 1 & 3 & 17 \\
III & 25 & 5 & 0 & 2 & 3 \\
Total & 75 & 30 & 3 & 7 & 20 \\
\hline
\end{tabular}

Foi possível determinar a associação entre LS e $\mathrm{T}$ positivos $(\mathrm{P}<0,0001)$, com $\mathrm{OR}_{\text {LS positivo } 42,8}$ (intervalo de confiança: 10,0 - 183,0). Como a tonsila é a primeira porta de entrada de microrganismos no animal, esperava-se que o número de amostras de tonsilas positivas fosse maior do que o de linfonodos submandibulares. A simples retirada das tonsilas da carcaça após o abate não eliminaria o risco de contaminação, mas poderia diminuí-lo em cerca de $30 \%$. O fato de linfonodos submandibulares estarem firmemente aderidos à musculatura da cabeça dificulta a sua retirada durante o processamento.

Berends et al. (1996) sugeriram a exclusão da carne da cabeça de suínos da fabricação de produtos de consumo como forma de evitar a contaminação. Entretanto, essa recomendação não é de fácil implementação na rotina de abate. Olsen et al. (2001) verificaram que a não remoção da língua e tecidos próximos, antes da retirada da cabeça, determinava decréscimo de $30 \%$ no número de carcaças positivas e consideraram essa medida importante para a redução de patógenos.

Foram identificados 14 sorovares diferentes em 101 animais positivos (Tab. 3). Apenas duas amostras não foram identificadas por não expressarem o antígeno flagelar. Os sorovares mais encontrados na etapa 1 foram Bredeney $(34,8 \%)$, Derby $(19,6 \%)$ e Typhimurium $(12,3 \%)$. O Bredeney foi o mais prevalente em estudo anterior realizado por Bessa et al. (2001) no mesmo frigorífico. Na etapa 2, o sorovar Typhimurium $(75,5 \%)$ foi o mais isolado $(\mathrm{P}<0,001)$, tanto nas tonsilas como nos linfonodos submandibulares.

Tabela 3. Número de amostras de Salmonella isoladas de conteúdo intestinal (CI), linfonodos mesentéricos (LM), pool de linfonodos submandibulares/ tonsilas (LT), linfonodos submandibulares (LS) e tonsilas (T) de suínos ao abate, segundo o sorovar

\begin{tabular}{|c|c|c|c|c|c|c|c|}
\hline \multirow{2}{*}{ Sorovar } & \multicolumn{5}{|c|}{ Isolados } & \multirow[b]{2}{*}{ Total } & \multirow[b]{2}{*}{$\%$} \\
\hline & $\mathrm{CI}$ & LM & LT & LS & $\mathrm{T}$ & & \\
\hline Agona & 1 & 2 & 1 & - & - & 4 & 2,2 \\
\hline Bredeney & 19 & 20 & 9 & 1 & 1 & 50 & 26,7 \\
\hline Derby & 12 & 10 & 5 & 1 & 2 & 30 & 16,0 \\
\hline Enteritidis & 2 & 5 & 1 & - & - & 8 & 4,3 \\
\hline Give & - & - & - & 1 & 1 & 2 & 1,1 \\
\hline Gold Coast & 1 & - & 2 & - & - & 3 & 1,6 \\
\hline Infantis & 3 & 5 & 4 & - & 1 & 13 & 7,0 \\
\hline Minnesota & - & 1 & 2 & - & - & 3 & 1,6 \\
\hline Montevideo & 1 & - & - & - & - & 1 & 0,5 \\
\hline Orion & 1 & - & - & - & - & 1 & 0,5 \\
\hline Panama & 3 & 1 & 1 & 1 & 3 & 9 & 4,8 \\
\hline Saint- Paul & - & - & 1 & - & - & 1 & 0,5 \\
\hline Salmonella sp. & 1 & 1 & - & - & - & 2 & 1,1 \\
\hline Tennessee & 3 & 3 & - & - & - & 6 & 3,2 \\
\hline \multirow[t]{2}{*}{ Typhimurium } & 3 & 7 & 7 & 19 & 18 & 54 & 28,9 \\
\hline & 50 & 55 & 33 & 23 & 26 & 187 & 100,0 \\
\hline
\end{tabular}

O sorovar Typhimurium tem sido o mais isolado de suínos. A porcentagem dos outros sorovares encontrados variou segundo os diversos estudos (Baggensen et al., 1999; Stege et al., 2000; Swanenburg et al., 2001a). A diversidade de sorovares encontrados em uma mesma granja ou mesmo nas diferentes amostras coletadas de um mesmo animal, aspecto já relatado por Bessa et 
al. (2001) e Michael et al. (2002), também ocorreu neste estudo.

$\mathrm{Na}$ primeira etapa, em apenas sete animais dos 22 positivos para Salmonella nas três amostras, encontrou-se o mesmo sorovar. Os maiores índices de concordância dos mesmos sorovares encontrados no mesmo animal foram verificados entre amostras de CI e LM (19/37), seguidos de LT e CI (13/26) e LT e LM (9/26). Na segunda etapa, o sorovar Typhimurium foi isolado de todas as amostras (16/20) de animais positivos.

Das 186 amostras testadas, $164 \quad(88,1 \%)$ apresentaram resistência a pelo menos um antimicrobiano. $\mathrm{O}$ maior número de amostras resistentes foi com tetraciclina $(75,8 \%)$, sulfonamida $(61,8 \%)$ e estreptomicina $(55,9 \%)$. Quanto ao grupo das quinolonas, apenas uma amostra foi resistente à ciprofloxacina e sete ao ácido nalidíxico (Tab.4).

Tabela 4. Número de amostras de Salmonella sp. resistentes a antimicrobianos isoladas de suínos ao abate, segundo o sorovar

\begin{tabular}{|c|c|c|c|c|c|c|c|c|c|c|c|c|c|c|c|}
\hline Sorovar & $\mathrm{N}$ & $\mathrm{AC}$ & AM & AP & $\mathrm{CF}$ & $\mathrm{CP}$ & $\mathrm{CL}$ & ES & GE & NA & $\mathrm{NE}$ & SL & ST & TE & TB \\
\hline Agona & 3 & 1 & - & 2 & - & 1 & 2 & 2 & - & - & 2 & 2 & 2 & 3 & - \\
\hline Bredeney & 50 & 3 & - & 33 & - & - & 38 & 35 & 1 & 1 & 25 & 40 & 34 & 37 & 1 \\
\hline Derby & 30 & - & - & 3 & - & - & 2 & 24 & 1 & - & - & 27 & 8 & 30 & - \\
\hline Enteritidis & 8 & - & - & 1 & 1 & - & - & 5 & 4 & - & - & 2 & - & 3 & 6 \\
\hline Give & 2 & - & - & - & - & - & - & - & - & - & - & - & - & 1 & - \\
\hline Gold Coast & 3 & - & 1 & 2 & - & - & 1 & 1 & 1 & - & - & 3 & 1 & 1 & - \\
\hline Infantis & 13 & - & - & 3 & - & - & 3 & 5 & - & - & 1 & 4 & 5 & 4 & - \\
\hline Minnesota & 3 & - & - & 1 & - & - & 3 & 1 & 1 & - & - & 3 & - & 1 & - \\
\hline Montevideo & 1 & - & - & - & - & - & - & - & - & - & - & - & - & - & - \\
\hline Orion & 1 & - & - & 1 & - & - & 1 & 1 & - & - & 1 & 1 & - & 1 & - \\
\hline Panama & 9 & 1 & - & 4 & - & - & 3 & 3 & 1 & - & - & 6 & 3 & 2 & 2 \\
\hline Saint-paul & 1 & 1 & - & 1 & - & - & 1 & 1 & - & - & 1 & 1 & 1 & 1 & - \\
\hline Salmonella sp. & 2 & - & - & 1 & - & - & - & 2 & 1 & 1 & - & 1 & 2 & 2 & - \\
\hline Tennessee & 6 & - & 1 & 2 & - & - & 1 & 3 & - & - & - & 3 & 2 & 5 & - \\
\hline Typhimurium & 54 & - & 1 & 3 & - & - & 3 & 21 & 3 & 5 & 2 & 22 & 7 & 50 & 1 \\
\hline Total & 186 & 6 & 3 & 57 & 1 & 1 & 58 & 104 & 13 & 7 & 32 & 115 & 65 & 141 & 10 \\
\hline
\end{tabular}

Fedorka-Cray et al. (1999a), Bahnson e FedorkaCray (1999) e Harvey et al. (1999) encontraram resultados semelhantes e observaram que os maiores índices de resistência ocorreram com antimicrobianos disponíveis há mais tempo no mercado, e que eram comumente utilizados na terapêutica. Dessa forma, o alto número de amostras resistentes à tetraciclina observado neste estudo pode ser explicado pelo uso freqüente desse antimicrobiano. Estudos realizados por Bahnson e Fedorka-Cray (1999) demonstraram a relação existente entre a utilização de tetraciclina na fase final de crescimento e o isolamento de amostras de Salmonella sp. resistentes a esse antimicrobiano em suínos ao abate.

Setenta e três amostras $(39,2 \%)$ de Salmonella sp. apresentaram resistência a quatro ou mais antimicrobianos, sendo consideradas multi- resistentes. Entre os sorovares mais prevalentes, o Bredeney apresentou o maior número $(35,7 \%)$ de amostras multi-resistentes $(\mathrm{P}=0,001)$. Apenas nove $(30 \%)$ amostras do sorovar Derby e nove $(16,6 \%)$ do sorovar Typhimurium foram multiresistentes.

Alguns sorovares isolados de suínos, como Typhimurium, Derby e Agona, apresentam maior índice de resistência (Fedorka-Cray et al., 1999b). Entre estes, $S$. Typhimurium DT104 tem apresentado maior freqüência de amostras com perfil de multi-resistência, tornando um sério problema para a saúde animal e humana (Baggesen e Aarestrup, 1998; Nielsen et al., 1999). Esse fagotipo tem se caracterizado por um perfil de resistência à ampicilina, tetraciclina, estreptomicina, cloranfenicol e sulfonamidas (Threlfall et al., 1994). Das 54 amostras do sorovar Typhimurium isoladas no trabalho, 
nenhuma apresentou perfil de resistência semelhante ao encontrado para o fagotipo DT104. Entretanto, apenas a fagotipificação poderá esclarecer se o fagotipo DT104 não estava presente entre as amostras, ou se apresentava marcadores de resistência distintos nessa região.

\section{CONCLUSÕES}

Observou-se associação entre isolamento de Salmonella sp. em LT e presença da bactéria no trato intestinal de suínos ao abate. A presença de Salmonella sp. no trato gastrintestinal e linfonodos submandibulares/tonsilas são fatores de risco para a contaminação de partes da carcaça utilizadas na fabricação de embutidos.

\section{AGRADECIMENTOS}

Ao CNPq pelo auxílio financeiro. À Dra. Eliane Falavina dos Reis, Departamento de Bacteriologia, Instituto Oswaldo Cruz, pela sorotipificação das amostras.

\section{REFERÊNCIAS BIBLIOGRÁFICAS}

BAGGESEN, D.L.; AARESTRUP, F. Characterisation of recently emerged multiple antibiotic resistant Salmonella enterica serovar Typhimurium DT104 and other multiresistant phage types from Danish pig herds. Vet. Rec., v.143, p.95-97, 1998.

BAGGESEN, D.L; CHRISTENSEN, J.; NIELSEN, A.C. et al. Characterisation of Salmonella enterica isolated from swine herds in a cross-sectional study of the Danish swine production. In: PROCEEDINGS OF INTERNATIONAL SYMPOSIUM ON THE EPIDEMIOLOGY AND CONTROL OF SALMONELLA IN PORK, 3., 1999, Washington. Anais... Washington: North Caroline State University, 1999. p.237-239.

BAHNSON, B.P.; FEDORKA-CRAY, P.J. The association of antimicrobial resistance pattern and reported usage of antimicrobials in commercial growing pig production. In: PROCEEDINGS OF INTERNATIONAL SYMPOSIUM ON THE EPIDEMIOLOGY
AND CONTROL OF SALMONELLA IN PORK, 3., 1999, Washington. Anais... Washington: North Caroline State University, 1999. p.240241.

BARRY, A.L.; THORNSBERRY, C. Susceptibility test: Diffusion test procedures. In: Manual of clinical microbiology. 4.ed. Washington, D.C: ASM, 1985. p.978-987.

BERENDS, B.R.; URLINGS, A.P.; SNIJDERS, J.M.A. Identification and quantification of risk factors in animal management and transport regarding Salmonella spp. in pigs. Int. J. Food Microbiol., v.30, p.37-53, 1996.

BERENDS, B.R.; VAN KNAPEN, F.; SNIJDERS, J.M.A. et al. Identification and quantification of risk factors regarding Salmonella spp. on pork carcasses. Int. J. Food Microbiol., v.36, p.199-206, 1997.

BESSA, M.C.; COSTA, M.; CARDOSO, M. Prevalence of Salmonella $\mathrm{sp}$. in slaughtered pigs in Rio Grande do Sul. In: PROCEEDINGS OF INTERNATIONAL SYMPOSIUM ON THE EPIDEMIOLOGY AND CONTROL OF SALMONELLA IN PORK, 4., 2001, Leipzig. Anais... Leipzig: University of Leipzig, 2001. p.189-191.

BLAHA, T.H. Pre-harvest food safety as integral part of quality assurance systems in the pork chain from "stable to table". In: PROCEEDINGS OF INTERNATIONAL SYMPOSIUM ON THE EPIDEMIOLOGY AND CONTROL OF SALMONELLA IN PORK, 4., 2001, Leipzig. Anais... Leipzig: University of Leipzig, 2001. p. 7-13.

DI GUARDO, G.; FONTANELLI, G.; PANFILI, G. Occurrence of Salmonella in swine in the Latim Region (Central Italy) from 1980 to 1989: a retrospective study. Vet. Q., v.14, p.6265. 1992.

FEDORKA-CRAY, P.J. The connection between Salmonella, swine, and food safety. In: PROCEEDINGS OF SWINE CONFERENCE, 1996, Nebrasca. Anais... Nebrasca: Lincoln, 1996. p.25-45.

FEDORKA-CRAY, P.J.; BAHNSON, P.B.; LADELY, S.R. Antimicrobial resistance patterns of Salmonella isolates collected from slaughter age pigs. In: PROCEEDINGS OF INTERNATIONAL SYMPOSIUM ON THE EPIDEMIOLOGY AND CONTROL OF 
SALMONELLA IN PORK, 3., 1999a, Washington. Anais... Washington: North Caroline State University, 1999. p.245-247.

FEDORKA-CRAY, P.J.; PETERSEN, K.E.; DARGATZ, D.A. et al. National antimicrobial resistence monitoring system: results for swine. In: PROCEEDINGS OF INTERNATIONAL SYMPOSIUM ON THE EPIDEMIOLOGY AND CONTROL OF SALMONELLA IN PORK, 3., 1999b, Washington. Anais... Washington: North Caroline State University, 1999. p.248249.

HARVEY, R.B.; FARRINGTON, L.A.; BUCKLEY, S.A. et al. Epidemiological survey of antimicrobial-resistant Salmonella in marketage swine. In: PROCEEDINGS OF INTERNATIONAL SYMPOSIUM ON THE EPIDEMIOLOGY AND CONTROL OF SALMONELLA IN PORK, 3., 1999, Washington. Anais... Washington: North Caroline State University, 1999. p.252-256.

HOLT, J.G.; KRIEG, N.R.; SNEATH, P.H. et al. Bergey's manual of determinative bacteriology. 9.ed. Williams \& Wilkims, 1994. 787p.

HURD, H.S.; MCKEAN, J.D.; WESLEY, I.V. et al. The effect of lairage on Salmonella isolation from market swine. J. Food Protec., v.64, p.939944, 2001.

MICHAEL, G.; COSTA, M.; CARDOSO, M. Comparison of selenite cystine broth, tetrathionate broth and Rappaport-Vassiliadis broth for the recovery of Salmonella from swine feces. In: PROCEEDINGS OF INTERNATIONAL SYMPOSIUM ON THE EPIDEMIOLOGY AND CONTROL OF SALMONELLA IN PORK, 3., 1999, Washington. Anais... Washington: North Caroline State University, 1999. p.77-79.

MICHAEL, G.B.; SIMONETI, R.; CARDOSO, M. et al. Sorotipos de Salmonella isolados em uma propriedade de suínos de terminação no sul do Brasil. Cienc. Rural, v.32, p.525-527, 2002.

MORROW, W.E.M.; DAVIES, P.R.; SEE, T. et al. The prevalence of Salmonella spp. in feces on farm and in ceca at slaughter. In: PROCEEDINGS OF INTERNATIONAL PIG VETERINARY SOCIETY CONGRESS, 16., 2000, Melbourne. Anais... Melbourne: IPVS, 2000. p.207 (Resumo).
NIELSEN, B.; MOGELMOSE, V.; SORENSEN, L.L. et al. Tracing back multiresistant Salmonella Typhimurium DT104 from pork at the slaugtherhouse to a specific swine herd by strategical use of serology and culture. In: PROCEEDINGS OF INTERNATIONAL SYMPOSIUM ON THE EPIDEMIOLOGY AND CONTROL OF SALMONELLA IN PORK, 3., 1999, Washington. Anais... Washington: North Caroline State University, 1999. p.261263.

OLSEN, A.M.; JENSEN, T.; DAHL, J. et al. Reduction in level of Salmonella on swine carcasses after slaughter without splitting the head. In: PROCEEDINGS OF INTERNATIONAL SYMPOSIUM ON THE EPIDEMIOLOGY AND CONTROL OF SALMONELLA IN PORK, 4., 2001, Leipzig. Anais... Leipzig: University of Leipzig, 2001, p.124-126.

ROSTAGNO, M.H.; HURD, H.S.; MCKEAN, J.D. et al. Salmonella infection in market swine during pre-slaughter holding. In: PROCEEDINGS OF THE INTERNATIONAL PIG VETERINARY SOCIETY CONGRESS, 17., 2002, Ames. Anais... Ames: Iowa State University, 2002. v.1, p.149 (Resumo).

STEGE, H.; CHRISTENSEN, J.; NIELSEN, J.P. et al. Prevalence of subclinical Salmonella enterica infection in Danish finishing pig herds. Prev. Vet. Med., v.44, p.175-188, 2000.

SWANENBURG, M.; BERENDS, B.R.; URLINGS, H.A.P. et al. Epidemiological investigations into the sources of Salmonella contamination of pork. Berl. Munchn. Tierarztl. Wschr., v.114, p.356-359, 2001a.

SWANENBURG, M.; URLINGS, H.A.P.; KEUZENKAMP, D.A. et al. Salmonella in the lairage of pig slaughterhouses. J. Food Protec., v.64, p.12-16, $2001 \mathrm{~b}$.

THRELFALL, E.J.; FROST, J.A.; WARD, L.R. et al. Epidemic in cattle and humans of Salmonella Typhimurium DT104 with chromosomally integrated multiple drug resistance. Vet. Rec., v.134, p.577, 1994.

USER'S guide: statistical analysis system. Release 8.2. Cary, NC: SAS Institute, 1999. 